\title{
Las competencias en alimentación en el diseño curricular de Introducción a la Física.
}

\section{The competences of feeding in the curriculum design of Introduction to Physics.}

COMUNICACIÓN

\section{Damian Lampert}

Universidad Nacional de Quilmes, Argentina. Contacto: damian.lampert@unq.edu.ar

\author{
Silvia Porro \\ Universidad Nacional de Quilmes, Argentina. Contacto:sporro@unq.edu.ar
}

Recibido: agosto de 2021

Aceptado: octubre de 2021

\section{Resumen}

En esta investigación se llevó a cabo un análisis cualitativo con carácter exploratorio del Diseño Curricular (DC) de Introducción a la Física (IF), asignatura de 4to año secundario de la Provincia de Buenos Aires, sobre la presencia de los contenidos de alimentación que se incluyen en el mismo. Para ello, se utilizaron siete dimensiones sobre las competencias en alimentación: (1) los alimentos; (2) funcionamiento del cuerpo con respecto a la nutrición; (3) cocinar; (4) cultivar y elaborar alimentos; (5) comprar alimentos; (6) comer en compañía y (7) la actividad física y el descanso. En el DC de IF se encontraron temas relacionados con el metabolismo y la energía química de los alimentos, la lectura de etiquetas, la huella ecológica en relación a la producción de alimentos y el funcionamiento de una cocina solar. Estos temas se asocian con las dimensiones 1, 2, 3 y 4 de las competencias en alimentación. Esto demuestra que el tema de la alimentación se encuentra presente en el DC de IF y que, de acuerdo a como es presentado, su abordaje permite trabajar los contenidos disciplinares de la física desde un enfoque CTS (Ciencia, Tecnología y Sociedad).

Palabras clave: Competencias en Alimentación; Diseño Curricular; Introducción a la Física; Educación CTS.

\section{Abstract}


In this research, a qualitative exploratory analysis was carried out on the Curricular Design (CD) of Introduction to Physics (IP) about the presence of the food contents included in it. For this, seven dimensions on food competencies will beused: (1) food; (2) functioning of the body with respect to nutrition; (3) cook; (4) grow and process food; (5) buyfood; (6) eat in company and (7) physicalactivity and rest. In the IP DC, topics related to the metabolism and chemical energy of food, the reading of labels, the ecological foot print in relation to food production and the operation of a solar cooker were found. These themes are associated with dimensions 1, 2, 3 and 4 of food skills. This shows that the subject of food is present in the CD of IP and that, according to how it is presented, its approach allows working on the disciplinary contents of physics from a STS approach (Science, Technology and Society).

Keywords: Food Skills; Curricular design; Introduction to Physics, STS educatio.

\section{Introducción}

En la enseñanza de las ciencias y más aún en los libros de texto, es frecuente confundir la alimentación con la nutrición. En muchos casos, suelen aparecer como sinónimos para establecer los puntos de la enseñanza. Sin embargo, en el proceso de alimentación las personas seleccionan los alimentos para consumirlos, es decir, se trata de un proceso voluntario y consciente que va a depender de aspectos económicos, culturales y humanos que permitan identificar qué ingerir. En cambio el proceso de nutrición ocurre a nivel celular y es de forma involuntaria y continua (España, Cabello y Blanco, 2014).

En relación a la alimentación, es imprescindible trabajar aspectos de la ciencia y la tecnología que engloban a la producción de alimentos. La ciencia y la tecnología de los alimentos incluyen aspectos de física, química y microbiología, para garantizar la calidad de los productos.

La enseñanza de temas relacionados a la alimentación, de ahora en más Educación Alimentaria (EA), permiten desarrollar habilidades de toma de decisiones que tendrán repercusiones en la salud de las personas a la hora de consumir algún alimento. (Prieto, España y Martín, 2012).

Asimismo, la alimentación es un tema para la inclusión de la perspectiva CienciaTecnología-Sociedad en las diferentes asignaturas de ciencias naturales y sociales. La EA permite trabajar aspectos ambientales, anatomofisiológicos, económicos, socioculturales y tecnológicos entre otros, ya que todas las personas necesitamos de los alimentos para vivir. Se han llevado a cabo múltiples investigaciones en relación a la enseñanza de la física, la química y la biología, desde un enfoque CTS, a partir del tema de la alimentación; por ejemplo, el abordaje de transgénicos (Reis, Cruz y Silva, 2019), las tecnologías de conservación (Almeida, 2018), las Enfermedades Transmitidas por Alimentos (ETA) en 
diferentes espacios (Lampert, Leotta y Porro, 2019), las radiaciones en los alimentos (Duarte, 2019) y los factores ambientales, urbanísticos y socioculturales que rondan a las enfermedades transmitidas por el agua (Praconovo et al, 2020).

Existen multitud de definiciones acerca de la enseñanza de la alimentación. En algunos casos, incluso se menciona las competencias en alimentación. Las competencias, pueden definirse como conocimientos, habilidades o actitudes y atributos que convierten a una persona en competente para el desarrollo de una tarea o actividad (Arias, 2019).

En el campo de la EA, Cabello, España y Blanco (2016) indican que no hay un consenso sobre las denominaciones para los conocimientos y habilidades asociados a la EA. Cullerton, Vidgen y Gallegos (2012) utilizaron el término de alfabetización en alimentación (foodliteracy) en la cual se incluyen los siguientes dominios: acceso, comida, planificación y gestión, selección, origen de los alimentos, preparación y lenguaje. Por su parte Vanderkooy (2010) estableció las técnicas o destrezas básicas en alimentación (foodskills) entre las cuales se incluye el conocimiento general de los alimentos (etiqueta, seguridad alimentaria, nutrición, etc.), la planificación de la alimentación, la conceptualización de los alimentos, las técnicas mecánicas en los alimentos y la percepción de los alimentos. Asimismo, la Food Standards Agency (2009), utilizó el término de competencias en alimentación asociadas a los alimentos, la seguridad y salud de las dietas, la sensibilidad en la consumición responsable y el cocinado y manipulación de los alimentos.

La clasificación con la cual se trabajará en este artículo corresponde a la propuesta por España, Cabello y Blanco (2014) y que, luego, se amplió en detalle por Cabello, España y Blanco (2016). Los autores utilizaron el término competencias en alimentación porque se relaciona con los referentes curriculares analizados por los autores. En este sentido, se representan siete dimensiones de las competencias en alimentación que se detallan en la siguiente tabla:

\begin{tabular}{|l|l|}
\hline Dimensión & Descripción \\
\hline (1) Los Alimentos & $\begin{array}{l}\text { Se incluyela composición nutricional de los alimentos más } \\
\text { frecuentes en las dietas, las guías alimentarias (las } \\
\text { conocidas pirámides alimentarias, óvalos alimentarios o } \\
\text { círculos), la clasificación de los alimentos, la lectura del } \\
\text { etiquetado de los alimentos en términos informativos para } \\
\text { fomentar o limitar un determinado tipo de alimento, de } \\
\text { acuerdo a la lista de ingredientes y la información } \\
\text { nutricional. }\end{array}$ \\
\hline $\begin{array}{l}\text { (2) Funcionamiento } \\
\text { respecto a la } \\
\text { nutrición }\end{array}$ & $\begin{array}{l}\text { Se incluyen en esta dimensión los procesos fisiológicos } \\
\text { vinculados a la nutrición y como pueden afectar en la salud } \\
\text { diversos hábitos deconsumo. También se mencionan los } \\
\text { requerimientos nutricionales en diferentes estadios de la } \\
\text { vida, los factores asociados al comportamiento alimentario } \\
\text { y la importancia del mantenimiento de una dieta saludable y }\end{array}$ \\
\hline
\end{tabular}




\begin{tabular}{|c|c|}
\hline & $\begin{array}{l}\text { hábitos alimentarios regulares relacionados a la tradición, la } \\
\text { cultura y el medio geográfico. } \\
\text { Un punto interesante es que esta dimensión se centra en la } \\
\text { prevención de los riesgos derivados de la manipulación de } \\
\text { los alimentos, es decir, la contaminación de los alimentos } \\
\text { por microorganismos y los factores asociados a la falta de } \\
\text { higiene del personal, la cocción inadecuada y la } \\
\text { contaminación cruzada, entre otros. }\end{array}$ \\
\hline (3) Cocinar & $\begin{array}{l}\text { Aquí se incluyen las habilidades para cocinar los alimentos } \\
\text { de forma atractiva, en el tiempo disponible y disminuyendo } \\
\text { los desperdicios. De esta forma, se haría mención de los } \\
\text { procesamientos de los alimentos como pelar, picar, batir, } \\
\text { etc. También se engloban en esta dimensión los aspectos de } \\
\text { la seguridad alimentaria relacionados a conocer y aplicar } \\
\text { normas de higiene que permitan prevenir la contaminación, } \\
\text { el deterioro y la descomposición durante el almacenamiento } \\
\text { y el procesamiento de los alimentos. }\end{array}$ \\
\hline $\begin{array}{l}\text { (4) Cultivar } \\
\text { elaborar } \\
\text { alimentos }\end{array}$ & $\begin{array}{l}\text { En esta dimensión se incluyen las formas de cultivar } \\
\text { alimentos y las técnicas de elaboración. Es decir, se incluyen } \\
\text { habilidades como plantar, hacer crecer, cosechar, etc. } \\
\text { También se incluyen los procedimientos básicos para la } \\
\text { elaboración de yogures y mermeladas, y las técnicas } \\
\text { agrícolas básicas relacionadas a los huertos escolares y } \\
\text { granjas familiares, como así la tecnología de los alimentos. } \\
\text { Entre las actividades se menciona hacer pan, elaborar } \\
\text { salazones, secar alimentos, encurtir hortalizas, conservar } \\
\text { alimentos por tratamientos térmicos y otros procesos en que } \\
\text { intervienen métodos de conservación, como la refrigeración } \\
\text { y la congelación. }\end{array}$ \\
\hline $\begin{array}{l}\text { (5) Comprar } \\
\text { alimentos }\end{array}$ & $\begin{array}{l}\text { Esta dimensión se relaciona con las capacidades de comprar } \\
\text { y preparar alimentos en diferentes situaciones dentro del } \\
\text { hogar. Asimismo, se incluyen los aspectos relacionados con } \\
\text { la capacidad de presupuestar, la comercialización, la } \\
\text { publicidad y el marketing. De esta forma, se incluyen } \\
\text { aspectos de competencias básicas en asuntos de dinero } \\
\text { relacionadas con la adquisición de alimentos. }\end{array}$ \\
\hline $\begin{array}{l}\text { (6) Comer } \\
\text { compañía }\end{array}$ & $\begin{array}{l}\text { Se refiere al hecho de compartir con familiares y amistades } \\
\text { en lugar de hacerlo de forma individual. }\end{array}$ \\
\hline 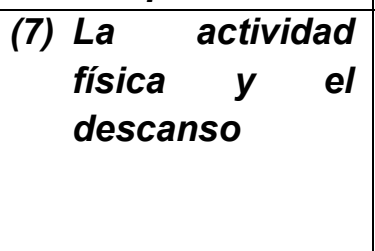 & $\begin{array}{l}\text { Esta dimensión se refiere a los aspectos relacionados con } \\
\text { la actividad física y la dieta para mantener un peso saludable. } \\
\text { Asimismo, se incluyen los aspectos relacionados con el } \\
\text { descanso adecuado como complemento de la alimentación } \\
\text { y la actividad física. }\end{array}$ \\
\hline
\end{tabular}

Tabla I. Dimensiones de las competencias en alimentación propuesta por España, Cabello y Blanco $(2014,2016)$. 


\section{La asignatura de Introducción a la Física}

Introducción a la Física (IF) es una asignatura de 4to año secundario de la Provincia de Buenos Aires, correspondiente a estudiantes de 15-16 años aproximadamente, de todas las orientaciones del ciclo superior. Los contenidos a trabajar, que se plantean en el Diseño Curricular (DC), se asocian con la conceptualización, transformación, transferencia, conservación y degradación de la energía. La asignatura se plantea como una herramienta para acercar la física al estudiantado a partir de la alfabetización científica.

Los ejes y núcleos de contenidos se presentan en la siguiente tabla:

\begin{tabular}{|l|l|}
\hline Ejes & Núcleos de Contenidos \\
\hline \multirow{2}{*}{ La energía en el mundo cotidiano } & Diferentes formas de energía. \\
\cline { 2 - 2 } & Formas utilizables de energía. \\
\hline \multirow{2}{*}{ La energía en el universo físico } & Generación natural de energía. \\
\cline { 2 - 2 } & $\begin{array}{l}\text { Energías macroscópicas y su } \\
\text { aprovechamiento. }\end{array}$ \\
\hline \multirow{2}{*}{ La energía eléctrica } & Generación y distribución. \\
\cline { 2 - 2 } & Usinas: potencia y rendimiento. \\
\hline \multirow{2}{*}{ La energía térmica } & Intercambios de energía. \\
\cline { 2 - 2 } & La energía y los seres vivos. \\
\hline \multirow{2}{*}{ La energía y la termodinámica } & Energía, calor y trabajo. \\
\cline { 2 - 2 } & Procesos reversibles e irreversibles. \\
\hline
\end{tabular}

Tabla II. Ejes y Núcleos de contenidos de IA (DGCyE, 2010).

Los alimentos constituyen una herramienta que permite trabajar aspectos de la energía. Martínez, Martínez, Martínez y Ruiz (2018) realizaron diferentes propuestas para abordar la física y la química en la cocina. Dentro del bloque de la energía, trabajaron la transmisión calorífica por conducción, convección y radiación, a partir de la cocción de la carne, con el objetivo de enseñarel efecto de la energía térmica sobre los alimentos (Martínez, Martínez, Martínez y Ruiz, 2018). Asimismo, utilizaron un capítulo del programa de televisión MasterChef, en el que se muestran diferentes dispositivos eléctricos para cocinar los alimentos y de esta forma, se propusieron analizar la conversión de la energía eléctrica en los diferentes dispositivos.

Este trabajo pretende realizar un análisis documental del DDC de Introducción a la Física, para conocer las competencias en alimentación que se presentan.

\section{Metodología}


Para llevar a cabo el análisis de las competencias en el DC, se utilizó una metodología cualitativa de indagación con carácter exploratorio que ya ha sido utilizada para el análisis documental de DC (Lampert y Porro, 2019). Asimismo, metodologías similares fueron utilizadas en otras investigaciones donde se determinaron las competencias en el ámbito de Alimentación y Actividad Física, a partir de un instrumento de análisis que engloba los contenidos competenciales que deberá adquirir el alumnado (Bayona, Garzón y Caurín, 2016). El análisis se llevó a cabo en dos etapas:

Etapa uno: análisis del DC y búsqueda de los términos asociados a la alimentación. Para el análisis del DC se trabajó con los apartados: mapa curricular, objetivos de enseñanza, objetivos de aprendizaje, organización de los contenidos, desarrollo de contenidos y orientaciones didácticas.

Etapa dos: clasificación de los resultados obtenidos en la etapa uno en función de las dimensiones propuestas por España, Cabello y Blanco (2014).

\section{Resultados}

A partir del análisis realizado, se encontró que dentro del eje "La energía en el mundo cotidiano" y el núcleo de contenidos "Diferentes formas de energía" se menciona el abordaje de la energía proporcionada por los alimentos y la información presentada en el envase de los mismos. EI DC, establece:

También podríamos preguntar a nuestros estudiantes, a partir de los datos proporcionados en los envases de alimentos, qué cantidad de fideos proveería la misma energía que una estufa en una hora. Esto nos da una pauta de los importantes consumos que tiene el organismo humano y de la gran concentración de energía que tienen los alimentos en un espacio relativamente reducido (DGCyE, 2010, p.20).

De acuerdo a lo mencionado, la propuesta para el trabajo áulico se relaciona con la dimensión de "Los alimentos" al mencionar el trabajo con las etiquetas y la energía química proporcionada por los alimentos lo cual muestra un caso concreto de educación CTS.

Dentro del eje "La energía térmica" y el núcleo "La energía y los seres vivos", se mencionan los contenidos relacionados con el metabolismo basal y la energía y alimentación. En relación a estos contenidos, se presenta el abordaje de los alimentos como fuente de energía química para las funciones fisiológicas esenciales, el trabajo muscular y la producción de calor para mantener la temperatura corporal. EI DC establece: "La energía disponible para la vida de todos los animales proviene de los alimentos consumidos. Esta energía química se utiliza en (...)" (DGCyE, 2010). 
De acuerdo a lo establecido por el DC, tanto en los contenidos como en el abordaje, aparece la dimensión de "Los alimentos" (al tratarse de la energía química de los mismos) y la dimensión de "Funcionamiento del cuerpo con respecto a la nutrición", ya que se tratan los aspectos bioquímicos de la alimentación, al mencionarse el metabolismo basal. Este concepto hace referencia a la energía necesaria para llevar a cabo las funciones vitales, como la respiración, la termorregulación, circulación sanguínea, etc. Para medirla la persona tiene que estar en reposo y con al menos 12 horas de ayuno, porque no se incluye la termogénesis inducida (energía que se gasta durante el proceso de digestión) por los alimentos en el metabolismo basal.

En el eje "La energía y la termodinámica", dentro del núcleo "Procesos reversibles e irreversibles" se menciona la huella ecológica asociada a la producción de alimentos. EI DC plantea:

Uno de los indicadores más difundido es la huella ecológica (...).El cálculo de la huella ecológica es complejo, y en algunos casos es imposible (...). Básicamente sus resultados están basados en la observación de los siguientes aspectos (...) Hectáreas necesarias para proporcionar el alimento vegetal necesario. Superficie necesaria para pastos que alimenten al ganado. Superficie marina necesaria para producir el pescado (DGCyE, 2010).

Siguiendo los lineamientos del DC y los aspectos relacionados a la huella ecológica, se menciona la dimensión sobre "Cultivar y elaborar alimentos" y los efectos ambientales que se podrían generar en relación a la producción de alimentos de origen vegetal y animal. Este punto es importante porque la huella ecológica es un recurso que permite evaluar el peso que se le brinda a los recursos energéticos y de esta forma, se genera un vínculo entre la ciencia, la tecnología y la sociedad.

Por último, en el apartado "Orientaciones didácticas", se propone el abordaje de investigaciones escolares en relación con los contenidos planteados en el DC. Entre uno de los ejemplos que menciona el DC, se encuentra:"¿Cómo puede construirse una cocina solar? ¿qué cantidad de alimento será posible cocinar en ella y en cuánto tiempo?” (DGCyE, 2010).

La propuesta mencionada puede trabajar diferentes aspectos de la energía como la transferencia por conducción, radiación y convección y las formas alternativas de energía como fuente para la tecnología de los alimentos. También, la propuesta puede generar la posibilidad de crear y desarrollar un secador solar de alimentos, en paralelo con la cocina. Estos lineamientos podrían concluir que la propuesta investigativa planteada por el DC se asocia con la dimensión de "Cocinar" de las competencias en alimentación.

A modo de resumen, la tabla III muestra lo obtenido en el análisis del DC y la asociación con las competencias en alimentación. 


\begin{tabular}{|c|c|c|c|}
\hline Eje & Núcleo & $\begin{array}{l}\text { Contenido } \\
\text { mencionado }\end{array}$ & $\begin{array}{l}\text { Competencias: } \\
\text { dimensión }\end{array}$ \\
\hline $\begin{array}{l}\text { La energía en el } \\
\text { mundo cotidiano }\end{array}$ & $\begin{array}{l}\text { Diferentes formas } \\
\text { de energía }\end{array}$ & $\begin{array}{lr}\text { Lectura } & \text { de } \\
\text { etiquetas y energía } \\
\text { química de los } \\
\text { alimentos }\end{array}$ & Los alimentos \\
\hline La energía térmica & $\begin{array}{l}\text { La energía y los } \\
\text { seres vivos }\end{array}$ & $\begin{array}{l}\text { Metabolismo } \\
\text { basal. Energía y } \\
\text { Alimentación }\end{array}$ & $\begin{array}{l}\text { Los Alimentos. } \\
\text { Funcionamiento } \\
\text { del cuerpo con } \\
\text { respecto a la } \\
\text { nutrición }\end{array}$ \\
\hline $\begin{array}{l}\text { La energía y la } \\
\text { termodinámica }\end{array}$ & \begin{tabular}{|l|} 
Procesos \\
reversibles \\
irreversibles
\end{tabular} & $\begin{array}{l}\text { Huella ecológica } \\
\text { en la producción } \\
\text { de alimentos } \\
\text { vegetales, } \\
\text { pescado y tierras } \\
\text { de uso para la } \\
\text { ganadería. }\end{array}$ & $\begin{array}{l}\text { Cultivar y elaborar } \\
\text { alimentos }\end{array}$ \\
\hline \multicolumn{2}{|c|}{$\begin{array}{l}\text { Propuesta de investigación transversal } \\
\text { para el abordaje de IF1 }\end{array}$} & $\begin{array}{l}\text { Creación de una } \\
\text { cocina solar }\end{array}$ & Cocinar \\
\hline
\end{tabular}

Tabla III. Relación entre los contenidos del DC y las competencias en alimentación.

\section{Conclusiones}

A partir del análisis realizado, es posible encontrar que se mencionan varios aspectos relacionados con los alimentos en el DC. La inclusión de contenidos de alimentos en la enseñanza de la física es un claro ejemplo que el objetivo de la asignatura es trabajar con los aspectos que rodean al estudiantado en su vida cotidiana y de esta forma, fomentar la alfabetización científica. Tal es así que la cocina solar, la huella ecológica y las etiquetas de los alimentos constituyen contenidos CTS para enseñar otros aspectos disciplinares de la física, como la transferencia, transformación y conservación de la energía.

En relación a las competencias en alimentación, de las siete dimensiones que establece la referencia utilizada, cuatro son incluidos en el DC. Lo cual, en complemento con otras asignaturas como Biología, Salud y Adolescencia e Introducción a la Química, permitiría favorecer el desarrollo de las competencias en alimentación en la escuela secundaria. Ya se han realizado estudios donde se encontró que los alimentos están presentes en las asignaturas anteriormente mencionadas (Lampert y Porro, 2019).

A pesar de los ejemplos y orientaciones establecidas por el DC, en los abordajes y propuestas se deja a consideración del profesorado la elección de temas de investigación y ejemplos para trabajar. De esta forma, se podrían incluir temas como equipos de

${ }^{1}$ No se incluye dentro de un Eje y Núcleo ya que en el DC no se detalle dentro de los mismos. 
refrigeración, para el eje de termodinámica; la irradiación de los alimentos, para las formas macroscópicas de energía y otros aspectos relacionados con la salud, la alimentación y la actividad física (de Eulate González, Cámara, Gavidia, Caurín y Martínez, 2015).

Como propuesta a futuro, se realizará un análisis de los libros de textos que se encuentran en el mercado sobre IA a fin de analizar qué ejemplos y cuál es el abordaje que realizan con respecto a los alimentos. También, si bien el DC establece estos lineamientos, es interesante conocer la voz del profesorado para saber si trabajan o no estos contenidos de alimentación.

\section{Referencias bibliográficas}

Almeida, M. P. D. (2018). Conservação de alimentos: uma proposta reflexiva para entrelaçar conhecimentos químicos e questões CTS (Master's thesis, Universidade Estadual de Maringá). Recuperado de: http://repositorio.uem.br:8080/jspui/handle/1/4690

Arias, J. L. I. (2019). La base teórica de las competencias en educación. Educere 23(74), 57-67.

Bayona, P., Garzón, A., y Caurín, C. (2016). Competencias en Alimentación y Actividad física. Análisis del currículo de la Educación Obligatoria. Los ocho ámbitos de la Educación para la Salud en la Escuela, pp. 129-149.

Cabello, A., España, E. y Blanco, A. (2016). La competencia en alimentación. España: Octaedro Recursos.

Cullerton, K.; Vidgen, H. y Gallegos, D. (2012). A review of food literacy interventions targeting dis advantaged young people. Recuperado de: http://eprints.qut.edu.au/53753/1/food literacy in-terventions review final.pdf.

De Eulate González, L. P., Cámara, E. L., Gavidia, V., Caurín, C., \& Martínez, M. J. (2015). ¿Qué enseñar en la educación obligatoria acerca de la alimentación y la actividad física? Un estudio con expertos. Enseñanza de las ciencias: revista de investigación y experiencias didácticas, pp. 85-100.

DGCyE. (2010). Diseño Curricular para la Educación Secundaria Ciclo Superior. ES4: Introducción a la Física / coordinado por Claudia Bracchi. - 1a ed. - La Plata: Dir. General de Cultura y Educación de la Provincia de Buenos Aires. Recuperado de: http://servicios.abc.gov.ar/lainstitucion/organismos/consejogeneral/disenioscurriculares/sec undaria/materias comunes a todas las orientaciones de 4anio/fisica 4.pdf

Duarte, N. (2019). Una propuesta didáctica para la enseñanza del concepto de energía nuclear mediante el uso de recursos educativos digitales con estudiantes de ingeniería (Tesis posgrado). Universidad Cooperativa de Colombia, Bogotá. Recuperado de: http://hdl.handle.net/20.500.12494/17389 
España, E., Cabello Garrido, A., y Blanco López, Á. (2014). La competencia en alimentación. Un marco de referencia para la educación obligatoria. Enseñanza de las ciencias: revista de investigación y experiencias didácticas, 32(3), 611-629.

Food Standards Agency (fsa) (2009). Users' guide. Secondary schoolaged materials (11 to 14 years and 16+ years). Recuperado de:

http://www.food.gov.uk/multimedia/pdfs/foodrouteuser2.pdf

Lampert, D. y Porro. S. (2019). ¿Se incluyen contenidos relacionados con las enfermedades transmitidas por alimentos en el nivel secundario de la provincia de Buenos Aires? Revista Química Viva, 3, (18). Recuperado de: http://www.quimicaviva.qb.fcen.uba.ar/v18n3/E0169.html

Lampert, D., Leotta, G. A., y Porro, S. (2019). Espacios con zoonosis y alimentos, una propuesta para la enseñanza CTS en Biología y Química. Educación en la Química, 25. Recuperado de: http://sedici.unlp.edu.ar/handle/10915/95949

Martínez, N. G., Martínez, S. G., Martínez, P. A., y Ruiz, L. A. (2018). Ciencia en la cocina. Una propuesta innovadora para enseñar Física y Química en educación secundaria. Enseñanza de las ciencias: revista de investigación y experiencias didácticas, 36(3), 179198.

Praconovo, Y., Lampert, D., Arango, C. y Porro, S. (2020). Las Enfermedades Transmitidas por el Agua desde la Geografía de la Salud: reflexiones y propuestas educativas. Trabajo aceptado para su publicación en el $3^{\circ}$ Congreso Internacional del Gran Chaco Americano llevado a cabo el 10, 11, 12 y 13 de noviembre de 2020 en Santago del Estero, República Argentina.

Prieto, T., España, E., y Martín, C. (2012). Algunas cuestiones relevantes en la enseñanza de las ciencias desde una perspectiva Ciencia-Tecnología-Sociedad. Revista Eureka sobre Enseñanza y Divulgación de las Ciencias, 9(1), pp. 71-77.

Reis, R. A. S., Cruz, L. P., y Silva, R. S. (2019). Alimentos Transgênicos: mediando a aprendizagem de alunos do ensino médios ob o enfoque CTS. Indagatio Didactica, 11(2), 621-636.

Vanderkooy, P. (2010). Foodskills of Waterloo Regionadults. Fireside Chat Presentation. 